\title{
Anomalous group velocity at the high energy range of real 3D photonic nanostructures
}

\author{
Muriel Botey ${ }^{*}$, Jordi Martorellb, ${ }^{\text {,a }}$, Gabriel Lozano ${ }^{\mathrm{c}}$, Hernán Míguez ${ }^{\mathrm{c}}$, Luís A. Dorado ${ }^{\mathrm{d}}$, Ricardo A. \\ Depine $^{\mathrm{d}}$ \\ ${ }^{a}$ Departament de Física i Enginyeria Nuclear, Universitat Politècnica de Catalunya, Barcelona, \\ Spain \\ ${ }^{b}$ ICFO-Institut de Ciències Fotòniques, Castelldefels (Barcelona), Spain \\ ${ }^{\mathrm{c}}$ Instituto de Ciencia de Materiales de Sevilla, Consejo Superior de Investigaciones Científicas, \\ Sevilla, Spain \\ ${ }^{\mathrm{d}}$ Departamento de Física, Facultad de Ciencias Exactas y Naturales, Universidad de Buenos Aires, \\ Buenos Aires, Argentina
}

\begin{abstract}
We perform a theoretical study on the group velocity for finite thin artificial opal slabs made of a reduced number of layers in the spectral range where the light wavelength is on the order of the lattice parameter. The vector KKR method including extinction allows us to evaluate the finite-size effects on light propagation in the $\Gamma L$ and $\Gamma X$ directions of fcc close-packed opal films made of dielectric spheres. The group is index determined from the phase delay introduced by the structure to the forwardly transmitted electric field. We show that for certain frequencies, light propagation can either be superluminal - positive or negative - or approach zero depending on the crystal size and absorption. Such anomalous behavior can be attributed to the finite character of the structure and provides confirmation of recently emerged experimental results.
\end{abstract}

Keywords: Photonic bandgap materials; Nanomaterials; Fast ligth.

\section{INTRODUCTION}

Systems that can either provide a reduction of the speed of light or superluminal light propagation have recently attracted significant attention since they could play a key role in the field of optical technology [1,2]. Among the variety of systems that hold such anomalous light propagation are two and three-dimensional (3D) periodically nanostructured materials [3]. In such systems, perfectly periodic calculations predict propagating modes with an almost vanishing slope, at the spectral range where the wavelength of light is on the order than the lattice constant or smaller. Such low dispersive modes are associated with strong group velocity reductions [3]. However, actual 3D systems do not seem to support such strong group velocity reductions and only exhibit a slowing down of two or three orders of magnitude $[4,5]$. Experiments have confirmed the existence of both behaviors in 3D ordered nanostructures, precisely in the high energy spectral range either in the $\Gamma L$ and $\Gamma X$ directions of fcc close-packed opal films [6,7]. In fact, interesting applications that take advantage of the special optical properties of periodic nanostructures at the high energy range, such as the anomalous group velocity reduction, have already been proposed to enhance various optical processes $[8,9,10,11]$. Besides, several experimental and theoretical works have already been devoted to explain the optical new features appearing at this energy range $[8,12,13,14]$.

In the present paper, we perform a comprehensive analysis on the influence of finite-size effects on the group velocity and group index of thin artificial opals, in a wide energy spectral range. As an illustration we consider a face-centeredcubic (fcc) close-packed opal made of polystyrene spheres (dielectric constant $\varepsilon_{s p h}=2.5$ ) in air $\left(\varepsilon_{a i r}=1\right)$. In the band structure such a perfect infinite system, in the $\Gamma L$ crystallographic direction, and for $1.05 \leq a / \lambda \leq 1.35$, the geometry of the structure gives rise to such high order extremely flat bands (see Fig. 1). In that energy region of almost vanishing dispersion, the group velocity of ideal infinite opals is expected to reach almost zero for a broad spectrum of wave vectors within the structure. Nevertheless, transitions from fast light to superluminal regimes with either positive or even 
negative group velocities have been observed in this same spectral region in high quality opals with controlled thickness [6]. On the other hand, in the $\Gamma \mathrm{X}$ orientation the slope of the photonic bands appears to be reduced at X high symmetry point. Precisely in the $\Gamma X$ direction, measurements evidence both slow light and fast light behaviors at $a / \lambda \sim 1.0$ and $a / \lambda \sim 1.3$, respectively [7]. In Fig. 1, the shaded regions show the high energy ranges where anomalous light propagation has been experimentally observed in opal films. In order to take full advantage of this new phenomenon for the control of the flow of light, we believe that this anomalous light propagation deserves a deeper analysis.
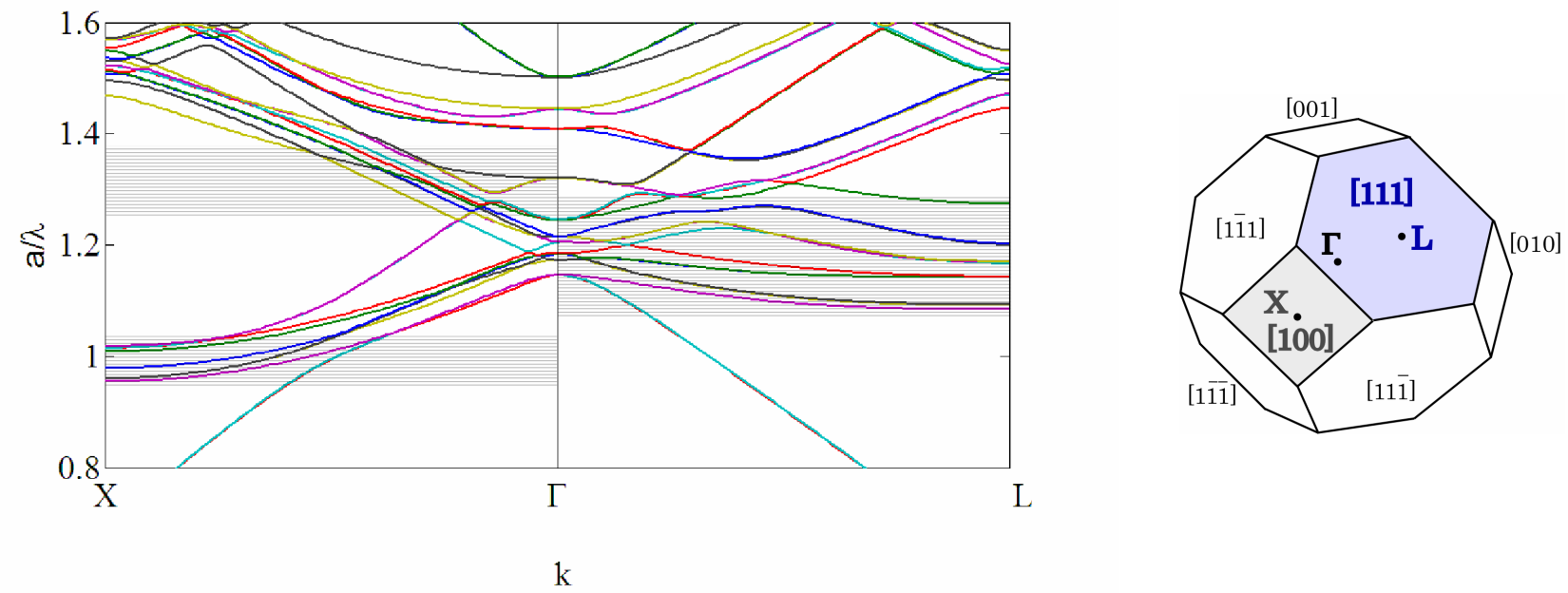

Figure 1. Left: High-energy band structure in the $\mathrm{X} \Gamma$ and $\Gamma \mathrm{L}$ crystallographic directions of an ideal close-packed fcc opal made of polystyrene spheres $\left(\varepsilon_{s p h}=2.5\right)$ in air $(\varepsilon=1)$. Calculations are performed by the plane-wave expansion method, using 729 plane waves [15]. Energy is expressed in reduced frequency units: lattice parameter, $a$, over wavelength in vacuum, $\lambda$. Right: First Brillouin Zone on a perfect fcc structure with the high symmetry points $\mathrm{X}$ and $\mathrm{L}$ and the [111] and [100] planes labeled.

Group velocity represents an important aspect to determine light propagation in real periodic structures. To this purpose we adopt the same code used in refs. $[8,12,13]$ which allows to consider a finite-width crystal slab taking into account the presence of a substrate. In the model, the effect of diffuse scattering due to the unavoidable structural imperfections is accounted by the introduction of an imaginary part in the dielectric constant of the spheres [12,22]. We are able to accurately reproduce the observed finite-size effects on group velocity in the $\Gamma L$ direction [6] and show that it may also be strongly affected by the presence of losses. Moreover, we theoretically explain the experimental results presented in ref. [7] which remained partially unexplained.

\section{GROUP VELOCITY DETERMINATION}

Recently, calculations based on the code reported by Stefanou et al. [16,17] developed from the vector Korringa-KohnRostoker (KKR) method [18,19] have provided very accurate predictions of the spectral dependence of transmittance, reflectance and intensity of the diffracted modes of opal films [12,13,20]. This approach is indicated to treat ordered assembles of spherical scatters and requires reduced computational effort. Moreover, it faces important experimental aspects such as the finite character of an actual crystal, the presence of interfaces, and the losses caused by the unavoidable crystal imperfections or dislocations. It has been shown that while disorder has little effect on the optical spectra in the low-energy range, it dramatically affects the response of the system in the high-energy range [12,21]. The KKR method refers propagation to a given set of $2 \mathrm{D}$ crystallographic planes and the fields at either side of the crystal are expressed using a plane wave expansion of the $2 \mathrm{D}$ reciprocal lattice vectors. Therefore, the relative amplitudes for the reflected and transmitted fields are denoted by $\mathrm{R}_{m l}$ and $\mathrm{T}_{m l}$, respectively; where the integers $(m l)$ refer to the reciprocal lattice vectors of the $2 \mathrm{D}$ plane. $\mathrm{T}_{00}$ represents the forwardly transmitted and $\mathrm{R}_{00}$ the specularly reflected electric fields, respectively. For convergence reasons, the values of the code parameter, LMAX, that controls the cut-off value of the angular momentum in the spherical-wave expansion of the electric fields is taken as LMAX $=9$. For all calculations 
shown in this paper, the code parameter, RMAX, of the plane wave expansion of the electric field, is taken as RMAX = 26; meaning that we use the first 41 reciprocal-lattice vectors [8,17,23].

In the $\Gamma L$ direction, diffraction in air starts, for the six lower order modes, at $a / \lambda \sim 1.63-$ while in glass at $a / \lambda \sim 1.07-$. For the next six modes diffraction starts at diffracted at $a / \lambda \sim 2.83$ in air $-a / \lambda \sim 1.85$ in glass - . The onset values are obtained from the condition of conservation of the tangential component of the wave vectors in a $2 \mathrm{D}$ close- packed triangular geometry:

$$
\frac{a}{\lambda} \geq \frac{\sqrt{2}}{n} \sqrt{1^{2}+\frac{(2 m+1)^{2}}{3}} .
$$

Thus, upon normal incidence on the [111] planes, and for $a / \lambda \leq 1.63$, the only reflected non-vanishing mode is the specular one, $\mathrm{R}_{00}$, see Fig. 2.a. Fig. 2.b shows that for the spectral range we are interested in, $a / \lambda \leq 1.4$, the forwardly transmitted, $\mathrm{T}_{00}$, field dominates transmittance even for a glass supported opal.
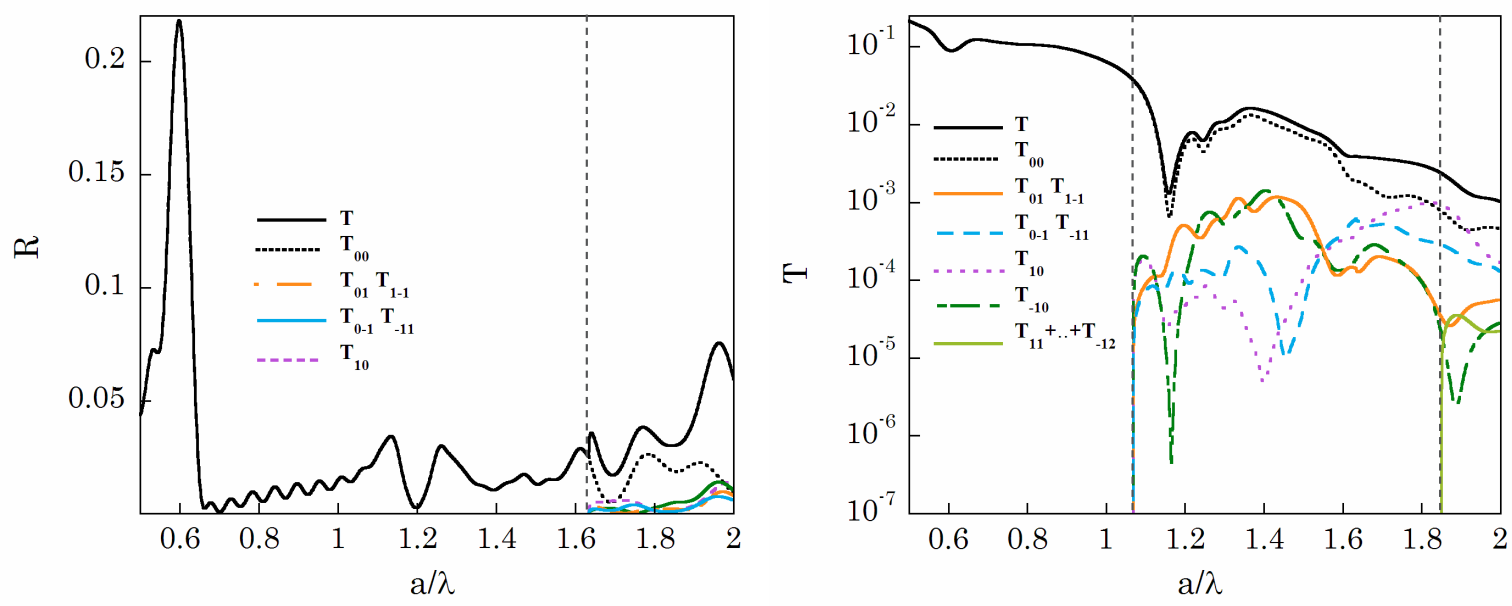

Figure 2. Calculated transmittance and reflectance in the $\Gamma L$ direction of a glass supported opal made of 10 [111] layers, taking $\varepsilon_{s p h}=2.5+0.1 \mathrm{i}$ for the dielectric spheres and $\varepsilon_{\mathrm{g}}=2.34$ for the glass substrate. a) Total reflectance, R,solid dark curve-, specular reflection coefficient, $\mathrm{R}_{00}$ - short-dashed dark curve - and first six diffracted modes with $m l=1-1,-11,10,-10,01,0-1$. b) Total transmittance, $\mathrm{T}$ - solid dark curve-, forward transmission coefficient, $\mathrm{T}_{00}$ - short-dashed dark curve-, individual contributions of the first six diffracted modes and total contribution of the next six higher order modes with $m l=-21,-12,2-1,1-2,11,-1-1$. The vertical dotted lines correspond to the diffraction onsets for the six lower order diffracted modes and the next six, respectively. All curves are plotted as a function of frequency in reduced, $a / \lambda$, units.

The condition of conservation of the tangential component of the wave vectors in a 2D square geometry reads:

$$
\frac{a}{\lambda} \geq \frac{\sqrt{2}}{n} \sqrt{1^{2}+m^{2}} .
$$

Form eq. (2) we obtain that, in the $\Gamma \mathrm{X}$ direction, diffraction in air starts at al $\lambda \sim 1.41-$ in glass at $a / \lambda \sim 0.91-$ for the four lower order modes, and at $a / \lambda \sim 2-$ in glass at $a / \lambda \sim 1.31-$ for the next four. Fig. 3.a shows how, upon normal incidence on the [111] planes, and for $a / \lambda \leq 1.41$, the only reflected non-vanishing mode is the specular one. Also in this direction, we can appreciate in Fig. 3.b that the forwardly transmitted mode, $\mathrm{T}_{00}$, dominates transmittance for $a / \lambda \leq 1.35$ even for a glass-supported opal.

Hence, despite the high complexity of the scattering phenomena that take place inside ordered nanostructures, the group velocity either in the $\Gamma \mathrm{X}$ or $\Gamma \mathrm{L}$ directions can be calculated from the forwardly transmitted fields, that propagate normal to the [111] and [100] planes respectively $[8,12]$. 


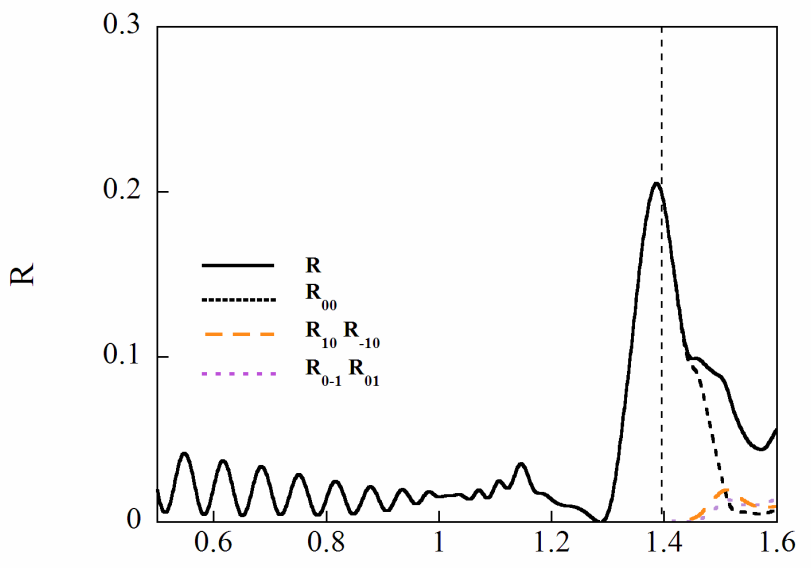

$\mathrm{a} / \lambda$

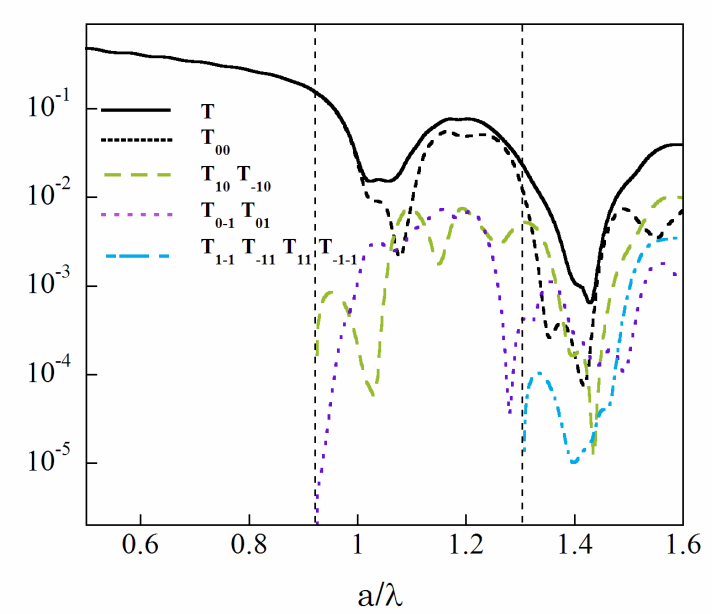

$\mathrm{a} / \lambda$

Figure 3. Calculated transmittance and reflectance in the $\Gamma \mathrm{X}$ direction of a glass supported opal made of 10 [100] layers, taking $\varepsilon_{s p h}=2.5+0.1 \mathrm{i}$ for the dielectric spheres and $\varepsilon_{g}=2.34$ for the glass substrate. a) Total reflectance, $\mathrm{R}$, - solid dark curve - , specular reflection coefficient, $\mathrm{R}_{00}$ - short-dashed dark curve — and first four diffracted modes with $m l=-10,10,01,0-1$. b) Total transmittance, T—-solid dark curve—, forward transmission coefficient, $\mathrm{T}_{00}$ - short-dashed dark curve-, contributions of the four lower order diffracted modes and next four diffracted modes with $m l=-11,1-1,-1-1,11$. The vertical dotted lines correspond to the diffraction onset for the four lower order diffracted modes and the next four, respectively. All curves are plotted as a function of frequency in reduced, $a / \lambda$, units.

For propagation in a given crystallographic direction, we obtain the group velocity from the phase delay, $\theta$, introduced by the structure to the forwardly transmitted field upon the incidence normal to a given plain ensemble. From such phase delay, we easily calculate the group index by means of the current definition:

$$
n_{g}=\frac{c}{v_{g}}=\frac{c}{L} \frac{\partial \theta}{\partial \omega}
$$

- where $L$ is the crystal thickness and $c$ the speed of light in vacuum-, to which the group velocity vg is inversely proportional. The frequency dependence of both, the phase delay introduced to the forwardly transmitted beam, and the group index, strongly depend on the index contrasts between the nanospheres and the surrounding medium [23]. While for very low dielectric contrasts the phase increases linearly with frequency, for higher index contrasts phase becomes a more complex function with either positive or negative slopes. This translates into an anomalous behavior of the group velocity, see Fig. 4.a and Fig. 4.b. Positive slopes of the phase profile tend to produce slow light, negative slopes fast light. Group index may attain exceptionally high values, greater than the values of refractive indices of the materials used, as well as superluminal negative values, becoming zero for certain frequencies.

\section{THICKNESS DEPENDENCE OF THE GROUP VELOCITY}

In the $\Gamma \mathrm{L}$ direction of an fcc lattice made of dielectric spheres in air, we obtain an anomalous phase behavior for light propagating at the high-energy range. Fig. 4.a displays the calculated phase delay introduced to the forwardly transmitted electric field where we can observe the phase fluctuations that give rise to an anomalous group velocity; the corresponding group index is plotted in Fig.4.b. We have considered that the effect of losses is weak and have taken a small imaginary part of the dielectric function $-\varepsilon_{s p h}=2.5+0.06 \mathrm{i}-$ which is in accordance with to the best fits of selfassembled samples $[12,21]$. If we investigate the range $1.05 \leq a / \lambda \leq 1.35$ we find three particularly interesting frequency regions, namely $a / \lambda \sim 1.15, a / \lambda \sim 1.25$ and $a / \lambda \sim 1.30$, see Fig. 4.a. For a given opal, at these three spectral positions, the phase delay introduced to the transmitted electric field does not show a linear dependence on the frequency but exhibits strong slope fluctuations. Since these spectral positions slightly depend on the actual crystal properties we will refer to them as 'A', 'B', and ' $\mathrm{C}$ ', respectively. Besides, the phase anomalous dispersion depends on the sample thickness, indicating a strong influence of the finite-size effects on the crystal properties. 

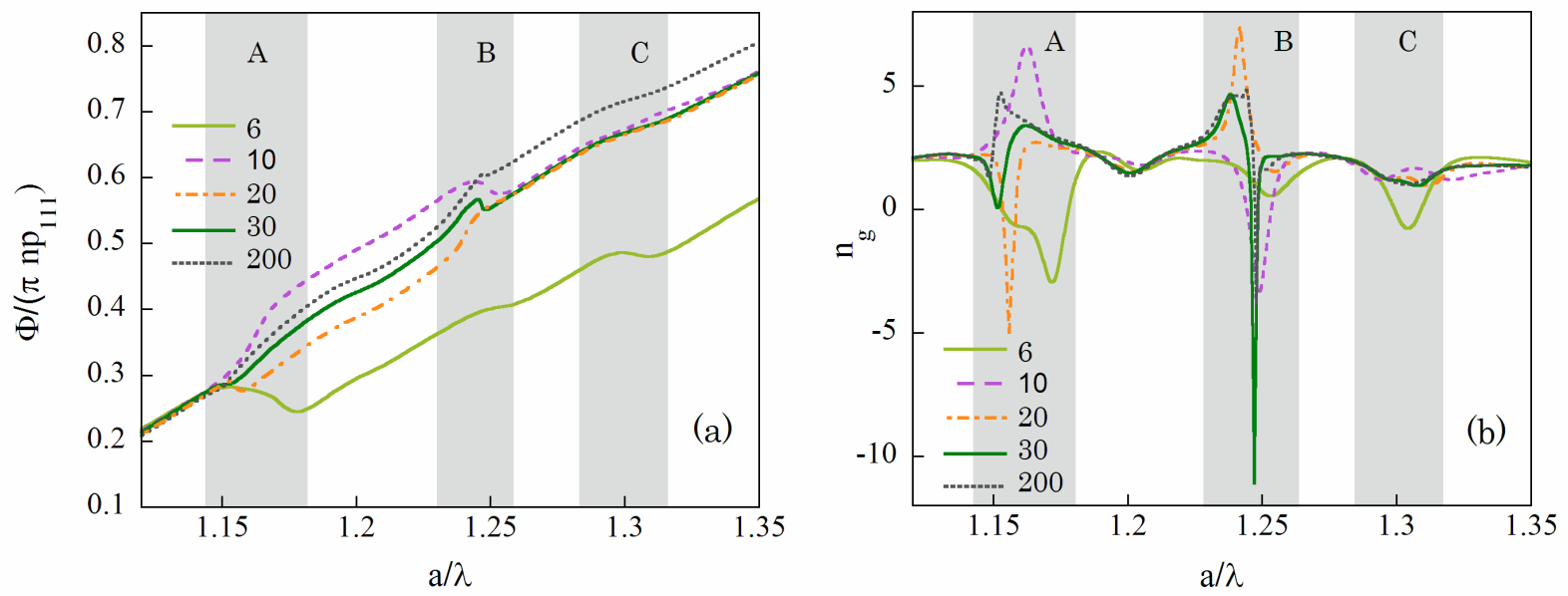

Figure 4. a) Phase delay divided by the number of [111] planes, in units of $\pi$, introduced to the forwardly transmitted field propagating in the $\Gamma \mathrm{L}$ of an fcc glass supported $\left(\varepsilon_{g}=2.34\right)$ structure. Nanospheres dielectric function: $\varepsilon_{s p h}=2.5+0.06 \mathrm{i}$. b) Corresponding group indexes for different opal thickness. The number of [111] planes is indicated on the figures inset. All curves are plotted as a function of frequency in reduced, $a / \lambda$, units.

Fig.4.b displays de corresponding group index dependence on thickness. A 6-[111]-planes-thick structure exhibits a highly negative group index value at the spectral position A and a positive small one at B; both corresponding to fastlight. Nevertheless, for a thickness of 10 [111] planes group index becomes negative at B, which still corresponds to fast light, while we find slow light at A. For a 20-[111]-layers-thick sample, the group velocity character changes: we recover fast light at A, but with negative group index, while negative fast light at B turns out to be positive fast light. Therefore, for a given thickness, adding only one layer introduces a flip of the phase slope. Such phase jump translates into a change of the group index sign, from a negative group index to a large positive group index. Taking for instance $\varepsilon_{s p h}=$ 2.5+0.06i, the first slope flip at A takes place between 8 and 9 [111] layers, and at B between 14 and 15 . Contrary to what could be expected, increasing the opal thickness does not cause the disappearance of the oscillations. As already mentioned, a second flip takes place at A between 21 and 22-[111]-layers and even a third change in the group velocity sign is observed at this spectral position. Also at B group velocity has a second sign flip between 29 and 30 [111] planes. Finally, for already thick systems, further increasing the number of [111] planes tends to smooth the fluctuations in this high-energy spectral range, either at A or B. Nevertheless, rather than giving rise to the expected broad fast light regime, the average group velocity reduction is small, except for very narrow resonances. In the case of the frequency range denoted by $\mathrm{C}$, and for the extinction here considered, group index is negative for thin opal films and a positive superluminal regime persists even for a 200-[111]-layers-thick structure.

The above predicted anomalous behavior of the phase delay was experimentally measured by Galisteo-López et al. at the frequency range A for a glass supported opal slab made of polystyrene spheres in air [6]. In that case, it was observed that the transition between negative superluminal light propagation to a low positive group velocity took place when the thickness was increased by a single [111] layer, from 7 to 8 . The difference in the phase delay introduced to the forwardly transmitted electric field between both samples, across the jump, was measured to be of $2 \pi$. Here we use the vector KKR method with extinction to calculate the phase difference occurring in this particular case in order to contrast our theoretical results with the experimental ones. The sphere's dielectric function is taken in accordance to polystyrene spheres dispersion [24] and a slight lattice relaxation [25]. As shown in Fig. 5.a, taking $\varepsilon_{g}=2.34$ for the glass substrate and no other adjusting parameter, the calculations give precisely a $2 \pi$ phase shift at the exact frequency where the flip of the group index sign takes place, in excellent agreement with the results reported in ref. [6]. We have also been able to reproduce with great accuracy the reported experimental results on group index. Our numerical results, for different opal thicknesses - keeping all other parameters constant - provide the group index dependence on thickness displayed in Fig. 5.b. Our predictions confirm theoretically that for an 8-[111]-layers-thick opal, group index inverts its sign from negative to positive, changing from superluminal to slow light propagation. 

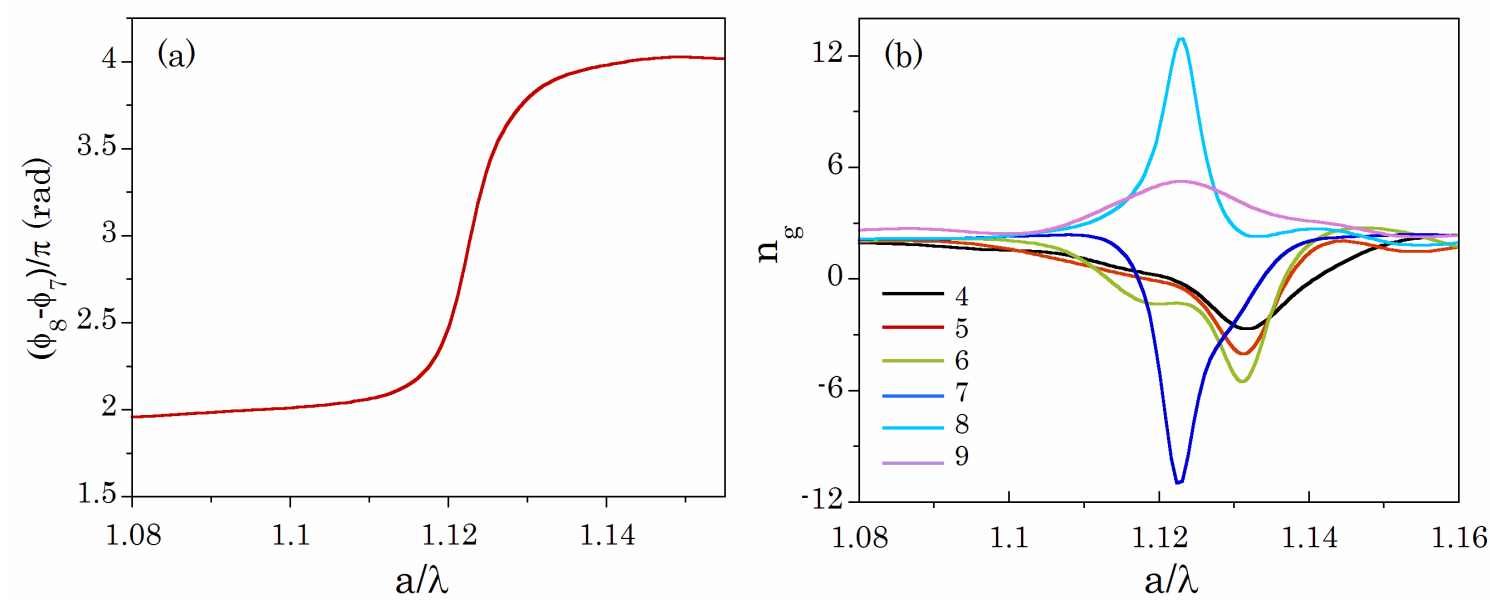

Figure 5. a) Difference phase delay introduced to the transmitted field between 8 and 7 [111] layers, in $\pi$ units. b) Calculated group index for different opal thickness as a function of reduced frequency in the A region. The graphic inset indicates to the number of [111] layers. The dielectric constant of the spheres and the glass support are $\varepsilon_{\text {shp }}=2.6+0.0575 \mathrm{i}$ and $\varepsilon_{\mathrm{g}}=2.34$, respectively [24,25].

In the GX crystallographic direction, the forwardly transmitted field exhibits also a complex light propagation behavior at the high-energy range. For $a / \lambda \leq 0.9$ the phase dependence on frequency - dispersion relation- is linear which corresponds an almost constant group velocity. Anyhow, as shown in Fig. 6.a, for higher frequencies we observe abrupt changes in slope which translate into an anomalous group velocity. The group index displayed in Fig. 6 explains the experimental observations of ref. [7] where two abrupt changes in the measured phase slope give two group index peaks.

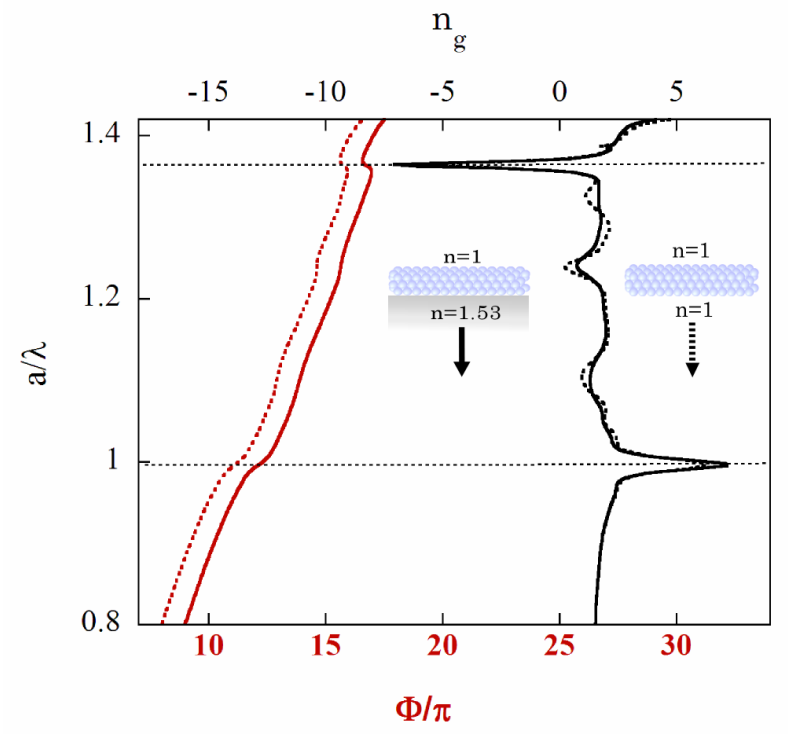

Figure 6. Phase delay, in units of $\pi$, introduced to a forwardly transmitted field propagating in the $\Gamma \mathrm{X}$ of a 7 [100]-layers glass supported opal — solid red curve — and an unsupported structure — dotted red curve —;curves are shifted for clarity. Calculated corresponding group index for the glass supported — solid black curve - and unsupported opal - dotted black curve- . The dielectric constant of the spheres and the glass support are $\varepsilon_{s h p}=2.5+0.06 i$ and $\varepsilon_{g}=2.34$, respectively. The vertical axis displays frequency in reduced, a/ $\lambda$, units.

A large positive group index is found at $a / \lambda \sim 1$ and a negative one at $a / \lambda \sim 1.35$. In order to reproduce this experimental observations we have used vector KKR with extinction, taking into account the presence of a glass substrate in the calculations. Notice however, that repeating the calculations for an unsupported opal provides almost the same results compare the dotted and solid curves on Fig.6. Hence, we believe that the origin of such phase fluctuations and the group 
index peaks is to be found in the collective electromagnetic resonances within the ordered array [13], which are affected by the presence of imperfections and finite-size effect.

\section{THE ROLE OF EXTINCTION}

Since disorder is present in any practical realization of periodic nanostructures, its effects are unavoidable specially at the high-energy range. Hence, we have also investigated the effect of losses caused by lattice imperfections or dislocations in the crystalline structure. For simplicity reasons we do not distinguish between different sources of disorder and losses. We have modeled extinction only by the introduction of an imaginary part to the sphere's dielectric function which accounts for the diffuse light scattering produced by imperfections instead of managing through the use of more than one parameter [21]. This method has already been successfully applied to colloidal crystals and validated with experimental data available in the literature $[12,15]$. We show that the group velocity does not only depend on the crystal geometry and dielectric contrast, but also the presence of imperfections play a crucial role.
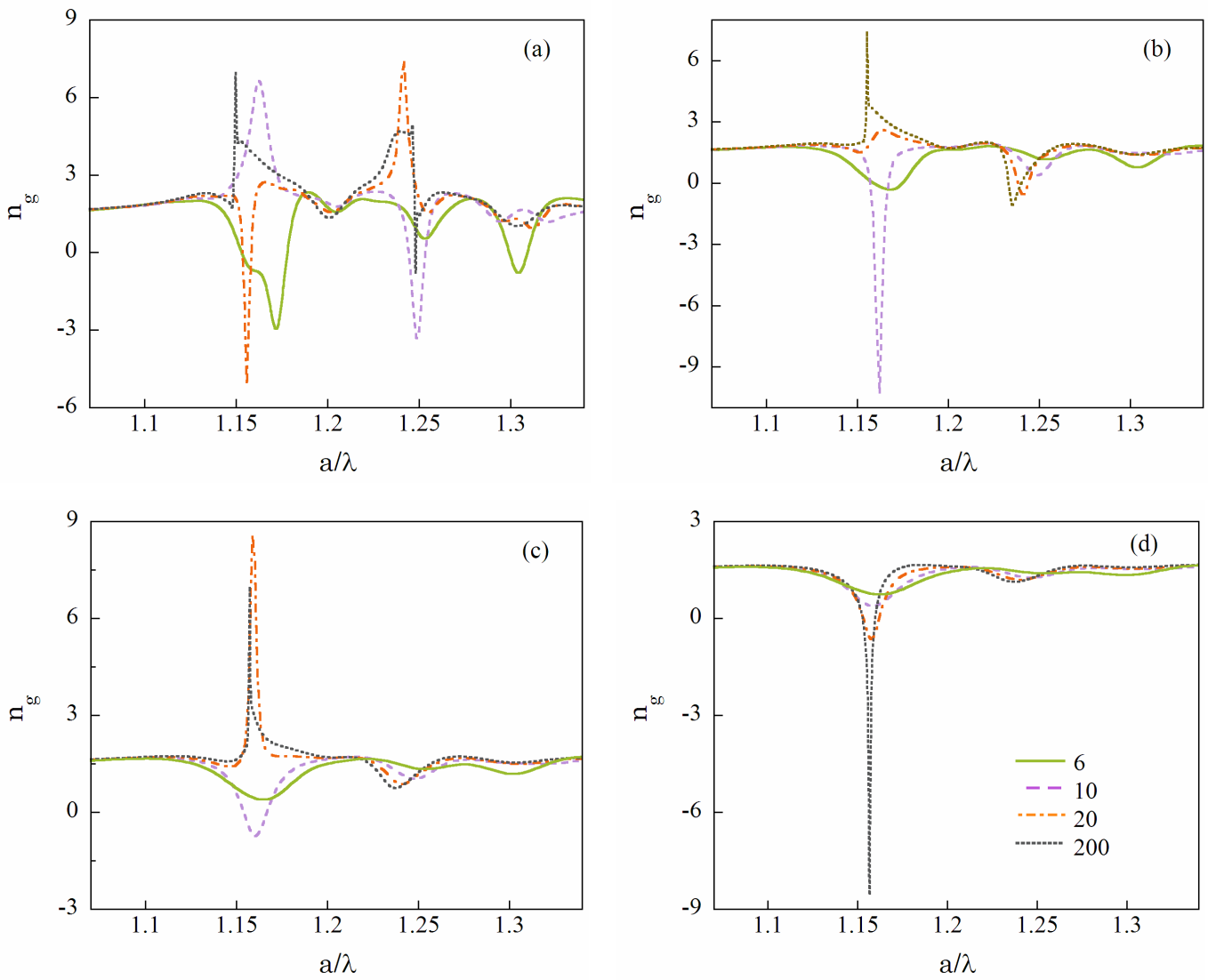

Figure 7. Group index in the $\Gamma \mathrm{L}$ direction of a glass supported opal made of an fcc lattice of polystyrene spheres in air, for different extinctions: (a) $\varepsilon_{\mathrm{sph}}=2.5+0.06 \mathrm{i}$, (b) $\varepsilon_{\mathrm{sph}}=2.5+0.10 \mathrm{i}$, (c) $\varepsilon_{\mathrm{sph}}=2.5+0.14 \mathrm{i}$, (d) $\varepsilon_{\mathrm{pph}}=2.5+0.18 \mathrm{i}$. The curves correspond to different opal thickness, in [111] planes: 6 (green solid curve), 10 (violet dashed curve), 20 (orange dashed-dotted curve), 200 (dotted black curve). All curves are plotted as a function of frequency in reduced, $a / \lambda$, units.

In the $\Gamma \mathrm{L}$ direction, the group index transitions from negative to positive values depend on extinction, following the description given above. This holds for any high quality artificial opal film not only at the spectral region A but also at B. The values normally required for the imaginary part of the dielectric constant to fit the reflectance spectra of real samples 
range from $0.04 \mathrm{i}$ to $0.14 \mathrm{i}[12]$. Fig. 7 shows how, for a given extinction the group velocity dependence on the opal thickness changes. In fact, increasing or decreasing such imaginary part of the dielectric function causes group velocity, at a certain frequency, to change its sign. For instance, for a 10-[111]-layers-thick opal group index is either positive or negative for 0.06i or 0.1i, respectively, see Fig. 7.a and Fig. 7.b. For higher extinctions, the group index fluctuations smooth out in general at B and C, see Fig. 7.c. At these spectral positions, for small losses group velocity can either be positive or negative, while for higher values it approaches zero, and finally all effects disappear and there is almost no anomalous propagation. On the other hand, at A, instead of a broad slow light regime there are only narrow resonances for certain absorptions and thickness. Moreover, it is important to note that for a sufficiently high absorption the superluminal character may persist even for thick samples made of over 200 [111] planes, see Fig. 7.d.

We have also seen that precisely for the case considered in Fig. 5, only a 10\% reduction, of the sphere's dielectric imaginary part, induces a sign flip of the phase between 6 and 7 crystal layers instead of taking place between 7 and 8 layers. This fact suggests that the lack of coincidence between the scattering matrix method prediction and the observations in ref. [6], could lie precisely on the role of losses.

A similar behavior is found when light propagates in the GX direction. For small absorptions the group index is constant for $a / \lambda \leq 0.9$, and exhibits strong fluctuations in the spectral range $0.9 \leq a / \lambda \leq 1.4$. While for $a / \lambda \leq 1.4$ increasing extinction group index oscillations tend to smooth, this is not the case for higher frequencies, where new resonances may appear increasing the sphere's dielectric imaginary part. The group index maximum and minimum observed in ref. [7] appear for extinctions over 0.14 . For lower extinctions a maximum instead of a minimum is found at $a / \lambda \sim 1.35$ which corresponds to slow light instead of fast light, while a less sharp minimum is found at $a / \lambda \sim 1.3$. Moreover, further increasing extinction the maximum of group index at $a / \lambda \sim 1$, becomes a minimum with a highly negative value. Hence, we observe, that also in this direction extinction plays an important role and may even be responsible for the group velocity character.

\section{CONCLUSIONS}

In summary we have theoretically obtained the group index of thin real opals from the phase delay introduced to the forwardly transmitted field, by using a vector KKR method with extinction. The results evidence nonconventional light propagation at the high-energy range of thin opal-like structures. In the $\Gamma \mathrm{L}$ crystallographic direction we have determined the presence of three frequency ranges with strong fluctuations of the group velocity. Not only is found the expected fast light regime but also a much richer optical behavior. Group velocity becomes either superluminical, positive or negative or approaches zero, depending on the crystal length. The above calculated group index describes very accurately previously reported experimental results for thin opal samples [6]. Also in the $\Gamma X$ direction, we have been able to explain the measured anomalies in the phase of ref. [7]. for thin artificial opals. We have shown that group velocity has not only strong finite-size effect dependence, but it also depends on extinction. For small absorptions, phase jumps and superluminal propagation persist even for a large number of planes, indicating that the finite character of the structure plays a crucial role even for relatively thick samples. Hence, it seems difficult to establish a direct identification between the above calculated anomalous group velocities for a real opal film with extinction and the modes dispersion of the band structure for an ideal infinitely periodic nanostructure. Moreover, lattice disorder, losses and finite-size effects may represent a limiting factor to the predicted attainable minimum group velocities at that energy range. Increasing the crystal thickness smoothes the group index profiles but does not give rise to a broad fast light regime and still some thin fast light resonances persist. Therefore, we have showed that at the high-energy range of a finite periodic structure, real aspects as imperfections and finite-size effects can no longer be ignored to predict light propagation. Although great effort has always been made to avoid structural imperfections in largely ordered nanostructures we find that precisely disorder and finite-size effects may give rise to interesting new optical properties. Such new features appearing at the high-energy range of 3D periodic structures could be advantageous for some applications such as a passive and compact optical delay line; they open new ways for the control of light propagation and could have a significant impact on the field of photonic technology.

\section{ACKNOWLEDGMENTS}

JM and MB acknowledge the Ministerio de Ciencia e Innovación, which supported the work under grants MAT200800910/NAN and CONSOLIDER NANOLIGHT CSD2007-00046. 
HM thanks the Spanish Ministry of Science and Innovation for funding provided under grants MAT2008-02166 and CONSOLIDER HOPE CSD2007-00007, as well as Junta de Andalucía for grant FQM3579. GL thanks the Spanish Research Council for funding of his scholarships and contracts under the JAE program

RAD acknowledges the support from the Consejo Nacional de Investigaciones Científicas y Técnicas (CONICET, Argentina) and the Agencia Nacional de Promoción Científica y Tecnológica (PICT-11--1785).

\section{REFERENCES}

1. Y. A. Vlasov, M. O'Boyle, H.F. Hamann, S. J. McNab, “Active control of slow light on a chip with photonic crystal waveguides," Nature 438, 65-69 (2005).

2. D. Dahan, G. Eisenstein, "Tunable all optical delay via slow and fast light propagation in a Raman assisted fiber optical parametric amplifier: a route to all optical buffering," Opt. Exp. 13, 6234-6249 (2005).

3. K. Sakoda, "Optical properties of photonic crystals," Springer-Verlag, Berlin (2005).

4. R. J. P. Engelen, D. Mori, T. Baba, L. Kuipers, "Two Regimes of Slow-Light Losses Revealed by Adiabatic Reduction of Group Velocity," Phys. Rev. Lett. 101, 103901 (2008).

5. J. G. Pedersen, S. Xiao, N. A. Mortensen, "Limits of slow light in photonic crystals," Phys. Rev. B 78, 153101 (2008).

6. J. F. Galisteo-López, M. Galli, A. Balestreri, L. C. Andreani, C. López, “Optical response of artificial opals oriented along the ГX direction," Appl. Phys. Lett. 90, 231112 (2007).

7. J. F. Galisteo-López, M. Galli, A. Balestreri, M. Patrini, L.C. Andreani, C. López, "Slow to superluminal light waves in thin 3D photonic crystals," Opt. Express 15, 15342 (2007).

8. M. Botey, M. Maymó, A. Molinos-Gómez, L. A. Dorado, R. A. Depine, G. Lozano, A. Mihi, H. Míguez, J. Martorell, "Second Harmonic Generation at the High Energy Range in a Nonlinear Opal Film," Opt. Express 17, 12210 (2009).

9. A. A. Fedyanin, O.Aktsipetrov, D.A. Kurdyukov, V.G. Golubev, M. Inoue, "Nonlinear diffraction and secondharmonic generation enhancement in silicon-opal photonic crystals," Appl. Phys. Lett. 87, 151111 (2005).

10. M. Scharrer, A. Yamilov, X. Wu, H. Cao, R. P. H. Changb, "Ultraviolet lasing in high-order bands of threedimensional ZnO photonic crystals,” Appl. Phys. Lett. 88, 201103 (2006).

11. H. Noh, M. Scharrer, M. A. Anderson, R. P. H. Chang, H. Cao, "Photoluminescence modification by a high-order photonic band with abnormal dispersion in ZnO inverse opal," Phys. Rev. B 77, 115136 (2008).

12. L. A. Dorado, R. A. Depine, G. Lozano, H. Míguez, "Interplay between crystal-size and disorder in the high-energy optical response of photonic crystals slabs," Phys. Rev. B 76, 245103 (2007).

13. L. A. Dorado, R. A. Depine, G. Lozano, H. Míguez, "Physical origin of the high energy optical response of three dimensional photonic crystals," Opt. Express 15, 17754 (2007).

14. M. Botey, L. A. Dorado, R. A. Depine, G. Lozano, H. Miguez, J. Martorell, "Anomalous group velocity in a 3D photonic nanostructure," in Conference on Lasers and Electro-Optics Europe/European Quantum Electronics Conference, Technical Digest (CD), (Optical Society of America, 2009), paper CK3.2Tue.

15. S.Guo. S.Guo, S. Albin, "Plane wave expansion method for photonic crystal calculations", Opt. Exp. 11, 167-175 (2002).

16. N. Stefanou, V. Karathanos, A. Modinos, "Scattering of electromagnetic waves by periodic structures," J. Phys.: Condens. Matter 4, 7389-7400 (1992).

17. N. Stefanou, V. Yannopapas, A. Modinos, "Heterostructures of photonic crystals: Frequency bands and transmission coefficients," Comp. Phys. Commun. 113, 49-77 (1998).

18. K. Ohtaka, "Scattering theory of low-energy photon diffraction," J. Phys. C: Solid State 13, 667-680 (1980).

19. A. Modinos, "Scattering of electromagnetic waves by a plane of spheres-formalism," Physica A 141, 575-588 (1987).

20. Luis A. Dorado, R. A. Depine, D. Schinca, G. Lozano, H. Míguez, "Experimental and theoretical analysis of the intensity of beams diffracted by three-dimensional photonic crystals," Phys. Rev. B 78, 075102 (2008).

21. L. A. Dorado and R. A. Depine, "Modeling of disorder effects and optical extinction in three-dimensional photonic crystals,” Phys. Rev. B 79, 045124 (2009).

22. M. Botey, M. Maymó, J. Martorell, "Band-structure determination for finite 3-D photonic crystals," Appl. Phys. B $81,277-281(2005)$. 
23. M. Botey, J. Martorell, L. A. Dorado, R. A. Depine, G.1 Lozano, H. Míguez, “Anomalous group velocity at the high energy range of a 3D photonic nanostructure," submitted to Opt. Exp.

24. X. Ma, J. Q Lu, R. S. Brock, K. M. Jacobs, P. Yang, X.-H. Hu, "Determination of complex refractive index of polystyrene microspheres from 370 to 1610 nm," Phys. in Med. and Bio. 48 4165-4172 (2003).

25. G. Lozano, L. A. Dorado, D. Schinca, R. A. Depine, H. Míguez,” Optical Analysis of the Fine Crystalline Structure of Artificial Opal Films," Langmuir 25, 12860-12864 (2009). 